Meta

Journal des traducteurs

Translators' Journal

\title{
Traduction et fixité idiomatique
}

\section{Salah Mejri}

Volume 55, numéro 1, mars 2010

Le parcours du sens : d'une langue à l'autre — Mélanges offerts à André Clas

The Way of Meaning: From a Language to Another - Collection of Articles Offered to André Clas

URI : https://id.erudit.org/iderudit/039600ar

DOI : https://doi.org/10.7202/039600ar

Aller au sommaire du numéro

\section{Éditeur(s)}

Les Presses de l'Université de Montréal

\section{ISSN}

0026-0452 (imprimé)

1492-1421 (numérique)

Découvrir la revue

Citer cet article

Mejri, S. (2010). Traduction et fixité idiomatique. Meta, 55(1), 31-41.

https://doi.org/10.7202/039600ar

\section{Résumé de l'article}

À la faveur des théories interprétatives, il n'est pas d'usage d'évoquer la dimension linguistique dans les traductions. Or, il y a un domaine où l'on ne peut en faire l'économie, celui où les énoncés à traduire impliquent des fixités linguistiques, c'est-à-dire des configurations spécifiques de la forme de la substance ou du contenu des signes linguistiques. Cela concerne des types de discours aussi variés que les séquences figées, les créations poétiques ou le discours humoristique. L'analyse des fixités linguistiques dans ces discours permet de constater que plus la stratification des fixités est importante, plus les difficultés de traduction sont complexes. Lors de la traduction de ces textes, on est le plus souvent appelé à faire des choix où l'on essaie de gérer au mieux les déficits lors du passage de $\mathrm{L}_{1}$ à $\mathrm{L}_{2}$. Toutes les illustrations concernent la traduction du français vers l'arabe. 


\title{
Traduction et fixité idiomatique
}

\author{
SALAH MEJRI ${ }^{1}$ \\ LDI (UMR 7187, CNRS) - Université Paris 13, Paris, France \\ TIL (00/UR/0201) - Université de la Manouba, La Manouba, Tunisie \\ smejri@ldi.univ-paris13.fr
}

\begin{abstract}
RÉSUMÉ
À la faveur des théories interprétatives, il n'est pas d'usage d'évoquer la dimension linguistique dans les traductions. Or, il y a un domaine où l'on ne peut en faire l'économie, celui où les énoncés à traduire impliquent des fixités linguistiques, c'est-à-dire des configurations spécifiques de la forme de la substance ou du contenu des signes linguistiques. Cela concerne des types de discours aussi variés que les séquences figées, les créations poétiques ou le discours humoristique. L'analyse des fixités linguistiques dans ces discours permet de constater que plus la stratification des fixités est importante, plus les difficultés de traduction sont complexes. Lors de la traduction de ces textes, on est le plus souvent appelé à faire des choix où l'on essaie de gérer au mieux les déficits lors du passage de $L_{1}$ à $L_{2}$. Toutes les illustrations concernent la traduction du français vers l'arabe.
\end{abstract}

\begin{abstract}
Thanks to interpretative theories, it is common practice not to evoke the linguistic dimension in translations. However, there is a domain where one cannot do without it, that is, where the utterances to be translated involve linguistic fixities, i.e., specific configurations of the form of the substance or the content of linguistic signs. That relates to types of discourses as varied as fixed phrases, poetic creations, humorous speech. An analysis of linguistic fixities in these discourses yields the conclusion that the more important the stratification of the fixities, the more complex the translation difficulties. During translation, one generally has to make choices in order to manage as well as possible the deficits in the passage from $L_{1}$ into $L_{2}$. All illustrations concern the translation from French into Arabic.
\end{abstract}

\section{MOTS-CLÉS/KEYWORDS}

fixité, déficit, humour, texte poétique, figement arabe-français

fixity, deficit, humour, poetic text, Arabic-French fixation

\section{Introduction}

La traduction de certains types de discours ne peut pas faire l'économie des spécificités idiomatiques. Même si l'on sait par ailleurs que les pratiques savantes de la traduction confirment le contraire. Ce constat se présente au traducteur qui négocie constamment les contenus à transmettre ou à garder lors du passage de $\mathrm{L}_{1}$ à $\mathrm{L}_{2}$ comme une évidence qui s'impose toutes les fois qu'on cherche à traduire des types particuliers de discours. Nous pensons tout particulièrement à tous les discours qui font de la fixité idiomatique une caractéristique conditionnant les contenus à traduire.

Nous préciserons ce que nous entendons par fixité idiomatique avant de fournir des exemples des types de discours à considérer et de démontrer en quoi ils présentent les mêmes difficultés devant le traducteur bien qu'ils appartiennent à des catégories qui semblent n'avoir aucun lien de parenté. 


\section{La fixité idiomatique}

Ceux qui travaillent sur la typologie des langues savent que certains mécanismes linguistiques généraux sont partagés par plusieurs langues que l'on regroupe le plus souvent en familles. Ces mécanismes qui concernent la répartition en parties du discours ou l'ordre des mots ou encore le figement sont souvent signalés comme étant des universaux de langue. Or, on sait par ailleurs que les invariants interlangues (Lazard 2006) sont des construits élaborés par les linguistes et qui échappent le plus souvent à des vérifications épistémologiquement contrôlables. La traduction et la comparaison des langues sont souvent utilisées, à cet effet, comme des outils à valeur heuristique.

Quand on pratique la traduction tout en ayant conscience du fonctionnement des langues, on est le plus souvent frappé par l'existence d'une forte solidarité entre certains contenus et la forme de leur expression dans les langues. Ces phénomènes sont regroupés couramment sous le qualificatif idiomatique, qui renvoie le plus souvent à ce qui est spécifique à chaque langue. De ce point de vue, le gallicisme serait un type de constructions idiomatiques propre au français. Or, force est de constater que l'idiomaticité (voir Mejri 2003) ne se limite pas à certaines constructions, mais dépasse de loin les phénomènes anecdotiques figurant dans les grammaires et les dictionnaires pour embrasser pratiquement tous les aspects de la langue.

Ce qui établit un lien entre tous les faits idiomatiques, c'est que les langues fixent leur matérialité (phonétique, syntaxique, lexicale, etc.) dans des contenus dont la forme de l'expression est tellement prégnante qu'il est vraiment difficile de dissocier les deux faces des signes linguistiques (forme du contenu et forme de l'expression). Cette solidarité entre les formes est trop stable dans la langue pour que le recours à un aspect puisse se faire sans le recours à l'autre. C'est ce que nous appelons fixité idiomatique.

Comme on sait que tout ce qui est fixe appelle la variation, on relève dans les différents types de discours des usages qui font de cette fixité leur terrain favori. Cela signifie que la créativité discursive puise dans le potentiel des langues constitué par cette fixité. On s'y est intéressé surtout en termes de détournement ou d'emplois ludiques. Or, à y bien réfléchir, on se rend compte que son champ d'action est beaucoup plus grand, puisqu'il relève de la création par transgression des règles. Si la créativité par l'application des règles s'inscrit à l'intérieur de la fixité de la langue, qu'il s'agisse de phonétique, morphologie, syntaxe ou sémantique, celle qui les transgresse remet en question cette fixité, la détourne et exploite tout le possible langagier qu'elle offre. Le produit de telles manipulations de la fixité, c'est tout discours qui tire son mode de réalisation dans le jeu sur la matérialité de la langue, c'est-à-dire la forme que celle-ci donne au contenu et à la substance. Nous présenterons dans ce qui suit des échantillons de ce genre de discours, en montrant à chaque fois en quoi ils mettent en jeu la forme et non la substance.

\section{Les réalisations discursives et la fixité idiomatique}

Nous nous limitons à deux échantillons de discours où se vérifie le jeu sur la forme linguistique: le discours poétique et le discours humoristique. 


\subsection{Le discours poétique}

À la base du discours poétique se trouve la fonction esthétique qui motive la création littéraire. Son objectif est la création du beau à partir de la matière linguistique. Le plus souvent, toutes les autres fonctions sont reléguées au second plan: ce n'est pas le contenu qui l'emporte; tout est mis au service de la forme. Tout dans la langue est mobilisé pour créer de l'interdit; d'où un jeu qui défigure totalement le texte de départ. Le passage de Queneau intitulé Contrepèteries dans Exercices de style illustre bien ce jeu:

Un mour vers jidi, sur la flate-lorme autière d'un arrobus, je his un vomme au fou lort cong et à l'entrapeau chouré d'une tricelle fessé. Toudain, ce sype verpelle un intoisin qui lui parchait sur les mieds. Cuis il pourut vers une vlace pibre. Heux pleures tus dard, je le devis revant la sare Laint-Gazare en crain d'étouter les donseils d'un candy.

(Queneau 1947, cité par Thierry 2003: 94)

Dans ce texte, le plus important pour Queneau, c'est la manipulation du signifiant qui substitue à l'arbitraire du signe linguistique un autre arbitraire, celui qui est créé à partir de ce qu'on pourrait considérer comme le degré zéro de l'écriture, une nouvelle configuration qui sollicite l'interprétant pour qu'il découvre la logique de l'auteur. Queneau recourt à la contrepèterie qui consiste dans l'interversion de lettres ou de syllabes à l'initiale des mots:

(1) Un jour vers midi > Un mour vers jidi (interversion de lettres)

(2) Soudain, ce type interpelle un voisin > Toudain, ce sype verpelle un intoisin (interversion de lettres et de syllabes)

De tels assemblages ne donnent accès au sens que moyennant des clefs de décryptage. Mais la concaténation des assemblages de lettres ne prend la configuration de combinaisons que grâce aux outils syntaxiques comme la préposition, les déterminants, les pronoms, etc. qui demeurent la plupart du temps inchangés. Ce qui est touché dans cette opération, c'est l'intégrité des unités lexicales.

La même opération peut concerner des séquences plus faciles à reconnaître parce que le jeu, même s'il relève de la même technique, ne porte que sur un seul phénomène ou une seule lettre dans le cadre d'un seul mot. Balzac a beaucoup usé de ce procédé, cité par Navarro Domínguez, à qui nous empruntons les exemples ci-dessous:

Abondance de chiens (biens) ne nuit pas.

Il faut ourler (hurler) avec les loups.

Les extrêmes se bouchent (touchent).

Il faut savoir se traire (taire) à propos.

(cité dans Navarro Domínguez 2000: 57)

La liste est longue (voir Navarro Domínguez 2000 : 57-58 notamment). Chez le même auteur, le même énoncé parémique peut faire l'objet de plusieurs transformations (Navarro Domínguez 2000: 69):

(3) Plus on est de fous, plus on rit. > Plus on est debout, plus on rit; plus on est des fous, plus on prie; plus on est debout, plus on crie; plus on a des poux, plus on cuit.

La différence fondamentale avec les manipulations de Queneau, c'est que l'énoncé obtenu reste intelligible. C'est la preuve que le niveau de fixité ciblé par Balzac n'est 
pas le même que celui où se situe le jeu de Queneau. Alors que ce dernier cherche à désintégrer totalement la configuration du mot:

(4) jour $>$ mour midi $>$ jidi

Balzac, lui, préserve l'unité lexicale. Les substitutions se font toujours dans le cadre d'unités lexicales bien fixées dans la langue. Au lieu d'avoir comme résultat un assemblage comme mour ou jidi, on obtient d'autres unités:

(5) des fous $>$ debout

des fous $>$ de poux

on rit $>$ on prie

on rit $>$ on crie

on rit $>$ on cuit

La fixité se place ainsi au niveau de l'agencement syntagmatique. S'il y a incongruité, elle se situe dans la combinatoire interne de la séquence figée qu'est le proverbe plus on est de fous, plus on rit. L'une des propriétés des séquences figées, c'est le blocage des substitutions paradigmatiques. Le déblocage entraîne le défigement du proverbe.

Il arrive que la forme de la substance reste intacte et que le jeu ne concerne que celle du contenu comme l'illustre l'exemple suivant:

La femme du Windisch regarde dans la cour. «Il envoie des amitiés à Amélie. Pourquoi ne lui écrit-il pas lui-même?

C’est la seule phrase qu'il ait écrite, dit Windisch, la phrase précédée de P.-S.»

Il remet la lettre sur le réfrigérateur.

"Qu'est-ce que ça veut dire, P.-S.?»

Windisch hausse les épaules. "Autrefois ça voulait dire pur-sang. Ça doit être un message secret.»

La femme de Windisch est sur le pas de porte. «Voilà ce que c'est quand les enfants vont aux écoles», soupira-t-elle.

(Müller 1988: 84-85)

La forme P.-S. reste inchangée; ce qui change, c'est le contenu qu'on lui attribue. Au lieu de post-scriptum, on opte pour pur-sang.

On peut multiplier les exemples. Contentons-nous uniquement de ces quelques vers extrait du $L a c$, de Lamartine:

Ainsi, toujours poussés vers de nouveaux rivages,

Dans la nuit éternelle emportés sans retour,

Ne pourrons-nous jamais sur l'océan des âges

Jeter l'ancre un seul jour?

(Lamartine 1820)

où la créativité se situe à plusieurs niveaux de la concaténation des mots: la structuration syllabique, la rime, les assonances et les allitérations, c'est-à-dire à un niveau de fixité qui relève à la fois de la langue et des canons esthétiques.

\subsection{Le discours humoristique}

Le discours humoristique renferme des variétés qui dépassent le cadre de cette contribution. C'est pourquoi nous ne fournissons que les éléments définitoires qui 
intéressent notre problématique. Pour ce faire, nous prenons des exemples extraits de L'Os à Moelle:

Orateurs qui désirez attendrir et charmer votre auditoire, ne mangez jamais de viande hachée, ça rend la parole coupante.

Petite annonce:

Pièces de rechange pour animaux divers: œil-de-bœuf, $7 \mathrm{~F}$; queue-de-rat, $3 \mathrm{~F}$; piedde-biche, 9 F; tête-de-loup, $10 \mathrm{~F}$; bec-de-cane, $11 \mathrm{~F}$

Petite annonce:

Philatéliste distingué s'intéresserait à voix ayant beaux timbres. Maîtres-chanteurs s'abstenir.

Petite annonce:

Auteur dramatique échangerait pièce en 4 actes contre 3 pièces et une cuisine.

Petite annonce:

Souffrant d'insomnie, j'échangerais matelas de plume contre sommeil de plomb.

$\left(\right.$ Laclos 1963) ${ }^{4}$

On peut ramener toutes les manipulations à un seul principe : l'association syntagmatique d'unités lexicales qui se font écho l'une à l'autre. Cela se produit quand on fait des rapprochements qui remotivent le sens littéral des séquences figées:

(6) viande hachée/ parole coupante

(7) pièces de rechange pour animaux/ œil-de-bœuf (fenêtre, lucarne, nom de plante) queue-de-rat (lime, tabatière, etc.) pied-de-biche (levier, instrument de cellier, etc.) tête-de-loup (brosse) bec-de-cane (clou à crochet)

(8) sommeil de plomb/ matelas de plume

ou qui jouent sur la polysémie des unités monolexicales:

(9) philatéliste/ voix, timbres, (maîtres)-chanteurs

(10) pièce (en 4 actes)/ 3 pièces (et une cuisine)

Se trouve au cœur de ces exemples la problématique des jeux de mots, qui se décline selon des configurations diverses et variées (voir Foucault 1988). Nous nous contentons ici de ceux qui touchent les blagues. Dans sa typologie des blagues, Sfar (2008) fait la distinction entre les blagues linguistiques et les blagues pragmatiques. Parmi les premières, elle retient celles qui sont construites sur la base d'une anomalie sémantique:

Deux maris discutent:

«Ma belle-mère est un ange!»

«T'as de la chance, la mienne est encore en vie.»

d'une anomalie phonétique: 
Dans un hôtel se trouve une belle jeune fille. Un type commence à lui faire des propositions, et la fille répond: «Never!» Il répond: «Neuf heures! D’accord!»

(cité dans Sfar 2008: 93)

d'une anomalie sémantique:

Nicolas demande à un copain: «Qu'est ce que ça veut dire: “I don't know” ?» et l'autre répond: «Je ne sais pas!»

(cité dans Sfar 2008: 93)

et d'un figement:

Un fou fait des bonds au-dessus d'un plat de spaghettis: «Je saute un repas!» (cité dans Sfar 2008: 93)

Après des exemples de passages littéraires et d'exemples humoristiques, nous fournissons dans ce qui suit une synthèse des éléments de fixité rattachés à la créativité des fragments cités:

- une fixité qui touche à la concaténation des phonèmes (des lettres) dans le cadre du mot: ce genre de fixité sert de base à des manipulations donnant lieu soit à des assemblages de phonèmes (lettres) sans aucune signification soit à d'autres mots;

- une fixité touchant aux unités polylexicales: dans ce cas, c'est le caractère figé de l'unité qui est concerné; ce qui a pour conséquence le défigement;

- une fixité relative à la forme du contenu: cela concerne les jeux sur les contenus sémantiques, qui laissent intactes les formes. Il s'agit dans ce cas d'associations sémantiques ou de remotivations, etc.

\section{Fixité et difficultés de traduction}

Si la fixité est «une structuration sous-jacente à l'usage de la langue conditionnant d'une manière relativement prédictible l'emploi des unités linguistiques dans l'énoncé» (Mejri 2008: 5-6), le non-respect de cette fixité donne lieu à des énoncés obliques dont nous avons fourni quelques exemples. Nous essayerons dans ce qui suit de montrer comment se présentent les difficultés de traduction de tels énoncés.

Quand la fixité concerne la concaténation des phonèmes (des lettres), deux situations se présentent: ou bien on traduit le texte obtenu après restitution de l'ordre normal des lettres dans L1 et on applique le même jeu d'interversion; ou bien on cherche des correspondants en L2 et on essaie d'effectuer les mêmes opérations de substitution. Dans le premier cas, il s'agit d'une opération mécanique qu'on transpose d'une langue à une autre, ce qui donne en arabe pour le passage de Queneau le texte suivant qui se caractérise par le même mode cryptonymique. Pour comprendre (11), il faut restituer le (10):

$(10)$

$$
\begin{aligned}
& \text { في يوم من الأيام عند منتصف النهار، رأيت في الجزءء الملفي لحافلة رجلا طويل العنق يحمل قبّعة موشحة بخيط مغتول. }
\end{aligned}
$$

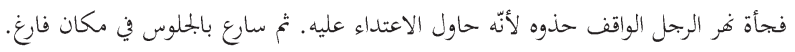

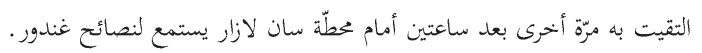


(11)

$$
\begin{aligned}
& \text { في موم من الامام عند ينتصف الرهار، لأيت في الخزء الجالفي لرافلة حجلا عويل الطنق يقمل حبعِ خوشحة بميط فنتول. }
\end{aligned}
$$

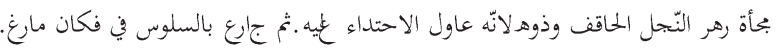

$$
\begin{aligned}
& \text { المقيت به ترة سخرى بعد أعثين امام سحطة مان سازار كلقع لِفضائح لندور. }
\end{aligned}
$$

Comme on le voit, sur le plan strictement technique, il est possible d'effectuer les mêmes opérations qu'en français:

(12) un jour vers midi $\rightarrow$ un mour vers jidi

$$
\begin{aligned}
& \text { في يوم من الأيام عند منتصف النّهار ـ في موم من الامام عند ينتصف الرهار / }
\end{aligned}
$$

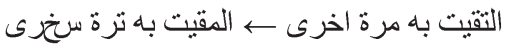

Reste à savoir si de telles pratiques sont courantes ou connues dans la langue d'arrivée. À notre connaissance, ce genre de manipulations n'est pas très usuel en arabe.

Dans le second cas de figure, la difficulté est plus grande parce qu'il ne s'agit pas d'une opération mécanique. La substitution d'un phénomène à un autre au niveau d'un mot dans un énoncé proverbial donne lieu à deux résultats: le défigement du proverbe et la réalisation d'une phrase libre ayant une double interprétation, celle suggérée par tous les éléments structurels et lexicaux qui rappellent le proverbe d'origine et celle de la phrase libre, fruit du défigement. Si on reprend les exemples déjà cités, on relève deux cas de figure:

- soit on trouve un équivalent en $\mathrm{L}_{2}$ et on porte la manipulation sur le même mot équivalent sans pour autant garantir les mêmes résultats au niveau de la signification de la phrase libre obtenue. Dans l'exemple français, les extrêmes se touchent $>$ les extrêmes se bouchent, il a suffit qu'on substitue $b$ - à $t$ - pour obtenir boucher au lieu de toucher et tout ce qui s'ensuit sur le plan sémantique. En arabe, on a l'équivalent (lit. les contraires se rencontrent), auquel on peut évidemment appliquer la même opération. Mais pour obtenir une phrase libre ayant du sens, on effectue une substitution qui touche deux phonèmes: (s'annulent) تنتفي (se rencontrent) تلثقي Il est clair que les extrêmes se bouchent n'a pas la même signification que les contraires s'annulent. S'il y a équivalence approximative, c'est grâce aux manipulations touchant au signifiant et au signifié;

- soit on limite la traduction à la manipulation: dans ce cas, à défaut d'un proverbe équivalent, on emploie n'importe quel proverbe et on lui applique la même opération. Ainsi, en arabe, هذا الثبل من ذاك الأسد (lit. ce lionceau de ce lion-là), qui est un équivalent mélioratif de Tel père, tel fils, peut donner lieu à : هذا الثبل من ذاك الك البلد (lit. ce lionceau de ce pays). Cette solution est relativement facile si on ne s'impose pas la contrainte de l'équivalence des significations des proverbes et des phrases obtenues dans les deux langues.

L'exemple de Herta Müller relatif à la siglaison est difficile à reproduire tel quel en arabe parce que ce mode de formation lexicale n'existe pas dans cette langue. Même si on le rencontre à l'écrit dans la désignation d'institutions ou de grade, par simple calque, il n'a jamais franchi l'étape de la graphie. C'est pourquoi il reste fondamentalement marginal. L'unique solution dans ce cas, c'est de trouver un équivalent 
cryptonymique à $P$.-S. On peut par exemple remplacer le sigle par un astérisque de renvoi à une note. Ainsi, on aurait le passage suivant:

«C'est la seule phrase qu'il ait écrite, dit Windisch, la phrase précédée de P.-S. ». Il remet la lettre sur le réfrigérateur.

"Qu'est-ce que ça veut dire, P.-S.?»

Windisch hausse les épaules. "Autrefois, ça voulait dire pur-sang. Ça doit être un message secret.»

$$
\begin{aligned}
& \text { قالّ فينديش : "إنّها الجملة الوحيدة التي كثبها، الجملة المسبوقة بنجمة". يرجع الرّسالة فوق }
\end{aligned}
$$

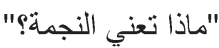

$$
\begin{aligned}
& \text {... "كانت تعني سابقا الثريّا. قد نكون هذه شفرة سريّة". }
\end{aligned}
$$

Comme l'astérisque est désigné en arabe par le terme étoile, la reprise se fera par un terme ayant le même sens en arabe mais moins usité ثرئيّ:raja].

La situation est beaucoup plus compliquée quand il s'agit de poésie. L’exemple de Lamartine présente l'avantage de s'inscrire dans des codifications bien établies relevant d'une fixité métrique qui s'ajoute à la fixité linguistique. La question est de savoir comment les traductions arabes disponibles rendent compte de ces deux types de fixité.

$$
\begin{aligned}
& \text { من شاطئ لشواطئ جدد } \\
& \text { يرمي بنا ليلّ من الأبد } \\
& \text { ما مرّ منه مضى لم يعد } \\
& \text { اهيهات مرسى يومه لغد }
\end{aligned}
$$

(Traduction de Ibrahim Néji)

$$
\text { تجري تجري الحكا أبدا تمضي أمانينا }
$$

(Traduction de Nicolas Fayadh)

$$
\text { كذلك لا نزال مدفوعين أبدا نحو شطآن جديدة، في الليل الأزلي، ومسافرين بلا عودة }
$$

(Traduction de Abdelaziz Sabri)

On se limite à ces trois traductions, même si plusieurs autres sont disponibles. Notre choix est motivé surtout par ceux effectués par les traducteurs. Plus on cherche à construire un équivalent rythmique et métrique en $\mathrm{L}_{2}$, plus on s'éloigne du texte en $\mathrm{L}_{2}$. La traduction de Abdelaziz Sabri est celle qui colle le plus au texte de Lamartine, 
mais ne tient pas compte de la dimension métrique. Pour celle de Nicolas Fayadh, on est dans la situation inverse: une structuration métrique arabe qui en fait un très joli poème, mais ne retient du poème français que la thématique générale. Pour celle de Ibrahim Néji, on a une solution intermédiaire. Ce qu'on peut retenir de telles pratiques traductionnelles, c'est que les solutions trouvées dépendent du nombre de fixités: plus les fixités sont nombreuses, plus on sacrifie d'aspects spécifiques au texte de $\mathrm{L}_{1}$.

C'est avec le texte humoristique que la superposition des fixités atteint toute son ampleur. Si on avait à fournir une caractéristique linguistique de ce genre, on dirait qu'il exploite au maximum les emplois limites ou obliques des mots dans le cadre de la phrase. La fonction ludique étant prédominante, tout dans l'énoncé humoristique est mis au service du jeu au détriment des autres fonctions du langage. Or, quand le jeu est strictement linguistique, la traduction s'avère très difficile, car il est rare que deux langues, en l'occurrence le français et l'arabe, offrent les mêmes possibilités d'emplois obliques pour les mêmes mots. Pour s'en convaincre, il suffit de procéder à une traduction littérale. La petite annonce suivante:

Auteur dramatique échangerait pièce en 4 actes contre 3 pièces et une cuisine.

$\left(\operatorname{Laclos} 1963^{4}\right)$

aurait la traduction suivante:

$$
\text { مؤلّف مسرحي يريد مبادلة مسرحيّة ب } 4 \text { فصول مقابل } 3 \text { بيوت و مطبخ }
$$

(Traduction de l'auteur)

Du moment que le pivot du jeu dans l'énoncé français est le mot pièce dans sa double signification de pièce de théâtre et de subdivision constitutive d'un habitat, et qu'en arabe, on dispose de deux mots différents pour renvoyer aux deux significations respectives, la traduction arabe détruirait complètement le jeu. Pour préserver le jeu sur la fixité linguistique à l'origine du jeu de mots, il faut trouver un équivalent en arabe qui soit le plus proche possible du texte français. C'est pourquoi on propose de remplacer auteur dramatique par poète, pièce (de théâtre) par vers (de poésie) et conserver pièce (logement). Cela permettrait d'avoir l'énoncé suivant:

$$
\text { شاعر يريد مبادلة قصيد و بيت بييت و مطبخ }
$$

(lit. poète échangerait un poème et un vers contre une pièce et une cuisine)

Le pivot du jeu serait ici le mot بيت [bajt] qui a la double signification de vers et pièce d'un logement. On notera qu'on a utilisé la forme du singulier بيت (un vers, une pièce), le pluriel étant différent pour chaque homonyme: بيات (des vers), بيوت (des pièces).

Comme on le constate, le cheminement fait du discours poétique au discours humoristique montre comment la question de la fixité linguistique est au cœur de la traduction. Traduire sans tenir compte des contraintes imposées par les langues concernées bloquerait les opérations de transfert qu’on cherche à réaliser avec la prise en compte de toutes les dimensions visées par le texte d'origine: littérarité, qualité esthétique, dimension ludique, etc. La complexité de la traduction est proportionnelle à la stratification des fixités impliquées par le texte de départ. La qualité de la traduction est elle aussi proportionnelle au nombre de fixités restituées en $\mathrm{L}_{2}$. 


\section{Pour ne pas conclure}

Nous avons voulu focaliser ici sur la dimension strictement linguistique, c'est-à-dire celle qui touche à la forme de la substance et du contenu, tout en sachant que l'opération de traduction ne se limite nullement à cet aspect. Notre souci n'est pas d'ignorer les autres dimensions mais d'isoler l'un des faits saillants imposés par les difficultés de traduction. Il reste évidemment des questions tout aussi pertinentes que la fixité linguistique. Nous en retenons tout particulièrement:

- celles qui relèvent des différentes stéréotypies, linguistiques et pragmatiques, rattachées d'une manière ou d'une autre aux langues impliquées dans la traduction;

- celles des univers de croyance et des différents mondes qui les structurent tels qu'ils s'expriment avec toutes sortes d'outils linguistiques;

- celles des croyances partagées et non partagées qui donnent matière aux usages obliques des langues.

Tant de questions mériteraient de plus amples développements.

\section{NOTES}

1. Ce travail a été réalisé dans le cadre du LIA-CNRS (Langues, traductions, apprentissage).

2. Müller, Herta (1988): L'homme est un grand faisan sur terre. Coll. Folio. Paris: Gallimard.

3. Lamartine, Alphonse de (1820): Le lac. In: Méditations poétiques. Consultée le 31 décembre 2009, <http://poesie.webnet.fr/lesgrandsclassiques/poemes/alphonse_de_lamartine/le_lac.html>.

4. Laclos, Michel (1963): L’Os à Moelle. Paris: Julliard.

\section{RÉFÉRENCES}

Ben Amor, Thouraya (2007): Le jeu de mots chez Raymond Queneau. Sousse: Publication de l'Université de Sousse, Faculté des lettres et des sciences humaines, Tunisie.

Buvet, Pierre-André (1998): Détermination et les classes d'objets. Langages. 131:91-102.

Cazeneuve, Jean (1996): Du calembour au mot d'esprit. Éditions du Rocher.

Foucault, Bruno de (1988): Les structures linguistiques de la genèse des jeux de mots. Coll. Sciences pour la communication. Vol. 23. Bern: Peter Lang.

FreI, Henri (1929) : La grammaire des fautes. Introduction à la linguistique fonctionnelle. Paris/ Genève, Guthner/Küdig.

Gross, Gaston (1996): Les expressions figées en français. Ophrys.

Gross, Gaston (1998): Pour une véritable fonction «synonymie» dans un traitement de texte. Langages. 131:103-114.

Guiraud, Pierre (1979): Les jeux de mots. Coll. Que sais-je?, n 165. Paris: PUF.

Guiraud, Pierre (1980): Typologie des Jeux de mots. Le Français dans le monde. 151:51-59.

LAzARD, Gilbert (2006): La quête des invariants interlangues. La linguistique est-elle une science? Paris: Champion.

Madini, Mongi, dir. (2002): 2000 ans de rire. Permanence et modernité. Actes du colloque

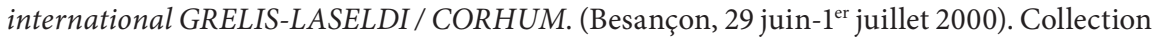
Annales littéraires de l’Université de Franche-Conté, 741, Linguistique et Sémiotique. Vol. 42. Besançon: Presses Universitaires Franc-comtoises.

Martin, Robert (1976): Antonymie, inférence et paraphrase. Paris: Klincksieck.

Martin, Robert (1987): Langage et croyance. Paris: Mardaga.

Mathieu-Colas, Michel (1998): Illustration d'une classe d'objets: les voies de communication. Langages. 131:77-90.

MejRI, Salah (1997): Défigement et jeux de mot. Études linguistiques. 3:75-92.

MejRI, Salah (2000): Traduction, poésie, figement et jeux de mots. Meta. 45(3):412-423. 
MejRi, Salah (2001a): Traduire les jeux de mots: repères méthodologiques. Mélanges offerts à Jean-Marie Van der Meerschen. Bruxelles: Les éditions du Hazard, 153-165.

MeJRI, Salah (2001b) : Norme et contre-normes: fonction identitaire et renouvellement du système. In: Actes du colloque "Diversité culturelle et linguistique: quelles normes pour le français?». Paris: Agence universitaire pour la francophonie, 67-73. Consulté le 10 janvier 2010, <http://www.auf.org/docs/1/normes-francais-2001-09.pdf>.

MejRi, Salah (2003): L'idiomaticité, problématique théorique. In: Salah Mejri, dir. L'espace euro-méditerranéen: une idiomaticité partagée. Tunis: CERES, 231-243.

Mejri, Salah (2004): Traduire les jeux de mots: approche linguistique. In: Actes du colloque "Traduire au 20 siècle» (Thessalonique, 27-29 septembre 2002). Université de Thessalonique / RLM.

Mejri, Salah (2006a): Polysémie, polylexicalité et jeux de mots. In: Martin Riegel, Pierre Swiggers et Catherine SCHNEDECKer, dir. Aux carrefours du sens - Hommages offerts à Georges Kleiber pour son $60^{e}$ anniversaire. Coll. Orbis / Supplementa. Louvain: Peeters, 683-695.

MejRI, Salah (2006b) : Polylexicalité, monolexicalité et double articulation : la problématique du mot. Cahiers de lexicologie. 89(2):209-221.

MejRI, Salah (2008): La traduction des jeux de mots. In: Salah MejRI, dir. Jeux de mots et traduction. Équivalences. 35:41-84.

Navarro Domínguez, Fernando (2000): Analyse du discours et des proverbes chez Balzac. Paris: L'Harmattan.

Sfar, Inès (2008): Traduire les blagues: jouer par/avec les mots. In: Salah MejRI, dir. Jeux de mots et traduction. Équivalences. 35:85-101.

Thierry, Jacqueline (2003): La traduction des jeux de mots. Paris: Presses Sorbonne Nouvelle.

Wieder, Catherine (2002): "L'esprit qui déshabille". Freud et le Witz. In: Mongi Madini, dir. 2000 ans de rire. Permanence et modernité. Actes du colloque international GRELISLASELDI / CORHUM. (Besançon, 29 juin-1 ${ }^{\text {er }}$ juillet 2000). Collection Annales littéraires de l’Université de Franche-Conté, 741, Linguistique et Sémiotique. Vol. 42. Besançon: Presses Universitaires Franc-comtoises, 111-119. 